\title{
Determination of sound velocities of "overcompressed" detonation in HMX-based explosive
}

\author{
Mikhail V. Zhernokletov, Alexey E. Kovaleva, Vladimir M. Bel'sky, and Evgeny N. Bogdanov \\ Russian Federal Nuclear Center - VNIIEF, Russia
}

\begin{abstract}
The authors present results of determination of sound velocities in explosion products (EP) of HMX-based explosive overcompressed up to the pressures of 50-85 GPa by overtaking unloading method. The radiowave and optical methods are used to record the time when a front of overcompressed detonation wave in investigated sample of high explosive (HE) is overtaken by expansion wave, which propagates from the back surface of impactor with sound velocity. The data on sound velocities, which were independently obtained by two different methods, were in agreement. The methods with use of radiointerferometer and indicator liquid are rather effective for determination of sound velocities in overcompresed EP and for investigation of parameters at the Jouget point of various HEs, which are required for calibration of their equations of state (EOS).
\end{abstract}

\section{Introduction}

When investigating explosives, to understand physics of detonation and formulate equations of state (EOS) of explosion products, plane-wave experiments are most often fulfilled. However, equilibrium thermodynamics is closely associated with rates of a chemical reaction in such experiments. In other words, to simulate and analyze experiments, there is a need to separate the data relative to rates of a reaction, from the data typical for equations of state. The values of sound velocities, measured with the same accuracy, as the values of velocities of detonation in plane-wave experiments with HE provide the determination of derivatives at a surface of an equation of state with the same accuracy, as the surfaces of EOS. The data on a sound velocity together with the Hugoniot adiabat for overcompressed detonation make it possible to calculate the Grueneisen coefficient and a pressure at the Chapman-Jouguet point (Ch-J). The $\mathrm{Ch}$ $\mathrm{J}$ pressure can be deduced with a good accuracy through thermodynamic state parameters, which are not connected with the effects of a reaction zone [1].

The overtaking unloading method with the use of indicator liquids for optical recording of thermal radiation caused by substance compression in a shock wave is the most informative method for measurement of sound velocities in metals compressed up to pressures higher $30 \mathrm{GPa}$. The current method is also used for measurements of sound velocities in explosive compositions (EC). In addition, it is possible to define a sound velocity in explosion products by using a radiointerferometer in the millimeter wavelength range.

The objective of the present work is to measure sound velocities in overcompressed explosion products of the HMX-based explosion composition containing a plastic bond made of fluoroplastic by using two independent

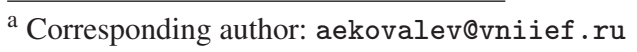

methods. A regime of overcompressed detonation is performed at the expense of loading of a "cold" composition with metal plates up to pressures of $100 \mathrm{GPa}$ in a shock adiabat.

\section{The rarefaction overtake technique for determination of sound velocity in explosion products}

The rarefaction overtake technique for determination of a sound velocity in substances is used to record the point of time when a front of an overcompressed detonation wave in the sample under study is overtaken by an rarefaction wave travelling from the back surface of an impactor with a sound velocity. Figure 1 presents the $X-t-$ diagram of flows occurring when a thin metal plate strikes the explosive charge with the thickness $\delta$.

Let us assume that a plate moves at the speed $W$ and "comes across" the explosive charge [2] at the point of time $t=0$ (point $\mathrm{O}$ ). While a plate impacts on HE, an overcompressed detonation wave is excited in HE, which propagates at the speed $D_{C}$. The shock wave OA is propagated through a plate at the speed relative to the fixed coordinate system $D_{l}-W$, which goes towards a free surface of a plate at the point $A$. A centered rarefaction wave arises at the point A, whose head characteristic arrives at the interface between a plate and explosion products at the point $\mathrm{B}$. An expansion wave begins to propagate in explosion products. The expansion wave overtakes a detonation front at the point $\mathrm{B}$, following which a front starts to slow down (see the insertion in Fig. 1).

In this case, there exist two ways for determination of an overtake moment (or a coordinate) of the detonation front by the help of a rarefaction wave in an explosive. The first way consists in the direct recording of detonation front movement in an explosive; a radio-wave technique 


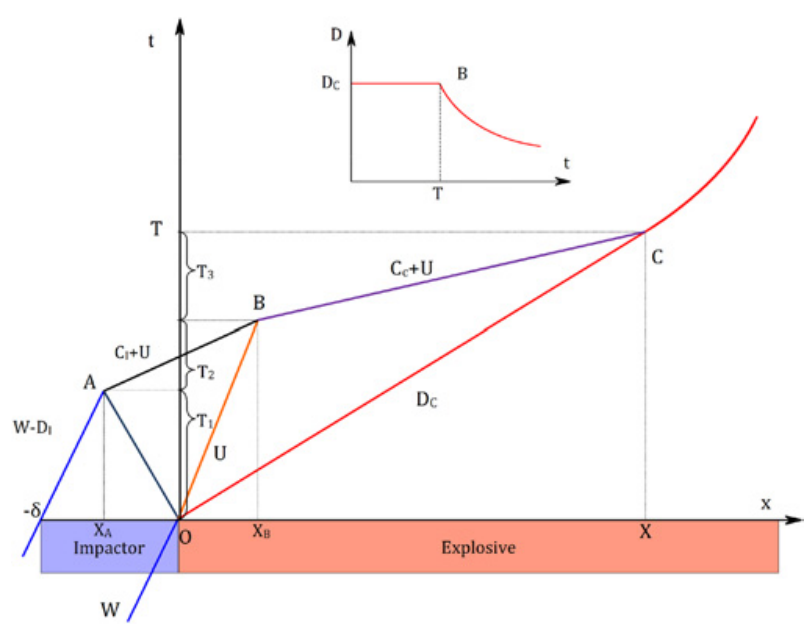

Figure 1. $X-t-$ diagram of impact of thin plate on explosive.

was used for this purpose in the present paper [3-5]. A thickness of an explosive sample decreases in the second way, and a layer of an indicator liquid is placed above the sample. The time when a rarefaction wave overtakes a shock wave front $[6,7]$ is recorded via the optical technique.

\subsection{Radio-wave technique}

To determine the arrival time of a longitudinal rarefaction wave $T$ into a detonation front in HE, we use the $X-T-$ diagram of a process in Fig. 1:

$$
T=T_{1}+T_{2}+T_{3},
$$

where $T_{1}$ is the time of a shock wave entering the back surface of a plate from a moment of impact; $T_{2}$ is the traveling time of a longitudinal rarefaction wave over a plate prior to its exit to the interface; $T_{3}$ is the traveling time of a longitudinal rarefaction wave in explosion products to a front of an overcompressed detonation wave.

Then:

$$
T_{1}=\frac{X_{A}}{W-D_{l}}=\frac{-\delta+W T_{1}}{W-D_{l}} .
$$

From which

$$
T_{1}=\frac{\delta}{D_{l}} .
$$

The time $T_{2}$ can be determined using the relation:

$$
T_{2}=\frac{x_{B}-x_{A}}{C_{l}+U},
$$

where $C_{l}$ is the velocity of a longitudinal rarefaction wave in impactor's material; is the velocity of the interface; $X_{A}=-\delta+W T_{1}, X_{B}=U\left(T_{1}+T_{2}\right)$ are the coordinates of the points $\mathrm{A}$ and $\mathrm{B}$ respectively.

Therefore

$$
T_{2}=\frac{\delta}{D_{l}} \cdot \frac{D_{l}-W+U}{C_{l}} .
$$

Evidently the value $T_{3}$ is defined as

$$
T_{3}=\frac{X_{B}-X_{B}}{U+C_{C}},
$$

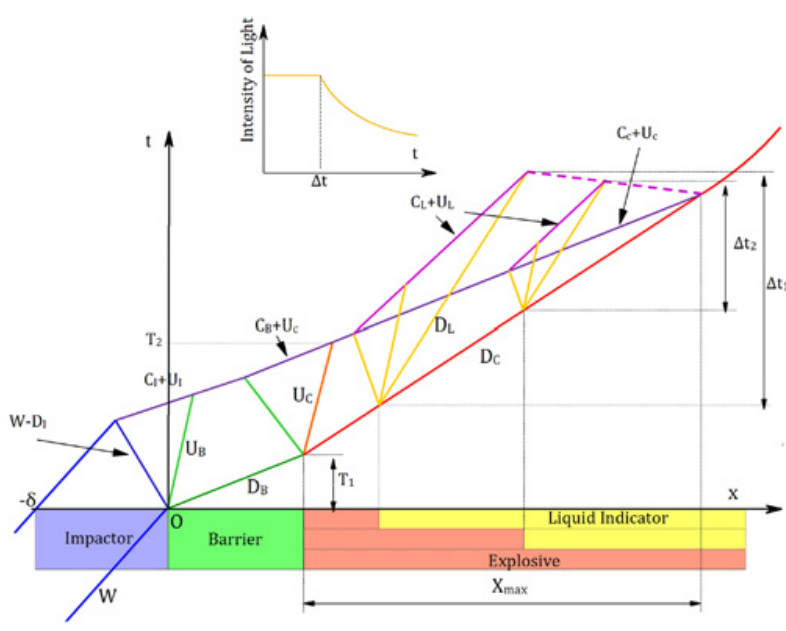

Figure 2. $X-t$ diagram of indicator technique.

where $X_{B}=D_{C}\left(T_{1}+T_{2}+T_{3}\right), C_{C}$ is the sound velocity in explosion products behind a detonation front in HE.

After a series of elementary transformations using relations (2)-(5) the formula can be obtained:

$$
T=\frac{\delta}{D_{l}} \cdot \frac{C_{C}}{C_{l}} \cdot \frac{C_{l}+D_{l}-W+U}{C_{C}-D_{C}+U} .
$$

Hence it follows the expression for a sound velocity in explosion products:

$$
C_{C}=\frac{D_{C}-U}{1+\frac{\delta}{T} \cdot \frac{C_{l}+D_{l}-W+U}{D_{l} C_{l}}} .
$$

To determine experimentally the sound velocity $C_{C}$ behind a front of an over compressed detonation wave in $\mathrm{HE}$ according to relation (7), it is necessary to record the instant of time $T$, after which a detonation wave velocity begins to decrease in HE (see the insertion in Fig. 1). The longitudinal sound velocity $C_{l}$ in impactor's material may be deduced as per a reference book (for example [8]) by using known state parameters at shock break out. In the case if a plate strikes a screen and the HE sample is placed behind a screen, the expression for the sound velocity $C_{C}$ is complicated a little.

\subsection{Optical technique}

The gist of the optical technique of measurement of sound velocities by using an indicator is presented in papers in detail $[6,7]$. Figure 2 demonstrates the $X-t-$ diagram of flows existing at the instant of time when a thin metal impactor strikes the system of screen-HE-indicator. Despite the more complicated character of processes in a system, the instant of the overtake of a detonation front is determined not in $\mathrm{HE}$ in the present technique, but in indicator liquid, which covers a sample made of HE under study. Constant or increasing radiation intensity is being recorded in the indicator prior to the moment of arrival of the first characteristic of a rarefaction wave at a shock wave front. Then radiation intensity begins to fall further in an indicator. 

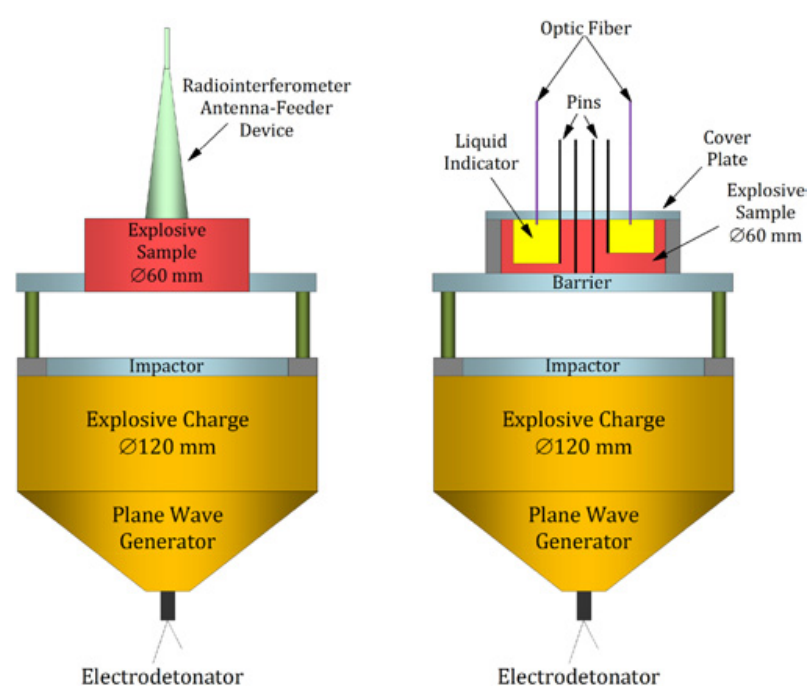

Figure 3. Sketches of experimental assemblies when using radiowave technique and optical technique of recording.

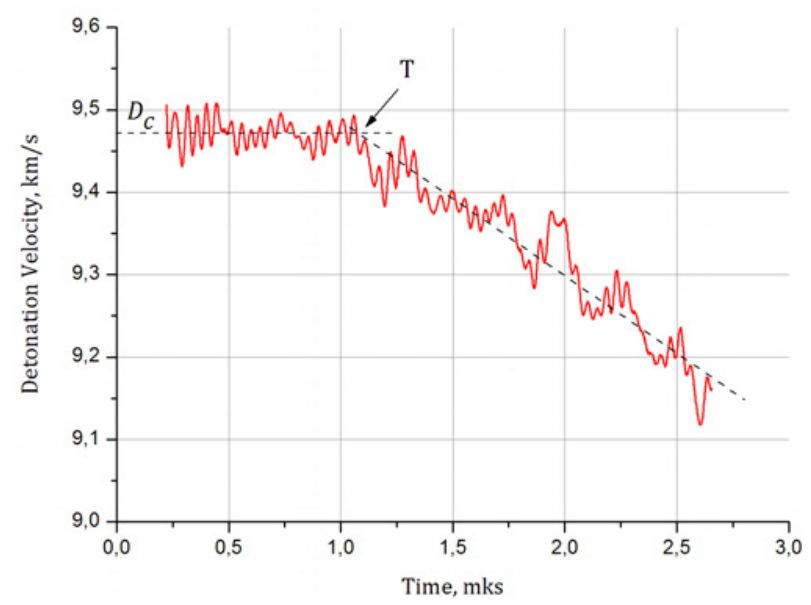

Figure 4. Dependence of overcompressed detonation wave velocity on time in sample.

The signal duration of indicator luminescence $\Delta t_{i}$ is determined for each first step of the sample $(i=1,2, \ldots n$, where $\mathrm{n}$ is the number of steps) in the experiment from the beginning of luminescence to the beginning of luminescence fall. The values $\Delta \mathrm{t}_{i}$ decrease linearly with increase in thicknesses $\Delta \mathrm{x}_{\mathrm{i}}$ of steps. This takes place always, independently of the relation of dynamic impedances of an indicator and a sample. A task consists in the determination of the sample thickness $X_{M A X}$ from the experiment; a rarefaction wave will overtake a shock wave front that moves ahead. A thickness of sample steps should be smaller than $X_{M A X}$. The $\mathrm{n}$ of independently measured points $\left(\Delta \mathrm{x}_{\mathrm{i}}, \Delta \mathrm{t}_{\mathrm{i}}\right)$ is recorded at the pressure $P$ in the sample in the experiment. By using these points the $a$ and $b$ coefficients of linear dependence are found through a method of linear approximation: $\Delta \mathrm{t}=a-b \Delta \mathrm{x}$. Then we obtain $X_{M A X}=a / b$ at $\Delta \mathrm{t} \rightarrow 0$ that is the sample's thickness, at whose interface an unloading wave will overtake a shock wave front (SWF) in a sample. The value of the sound velocity $C_{C}$ is determined in explosion

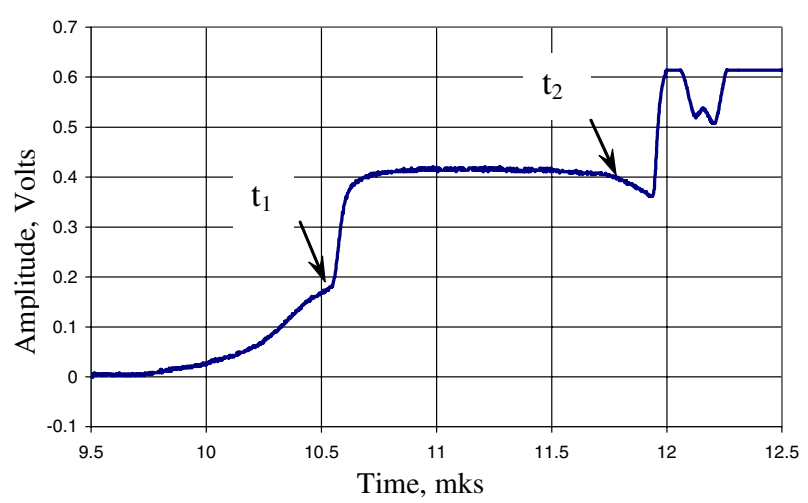

$\mathrm{t}_{1}$ - time of shock wave entering the indicator; $\mathrm{t}_{2}$ - time when the rarefaction wave overtakes the shock wave front.

Figure 5. Recording of luminescence of carbogal behind a step made of HE sample.

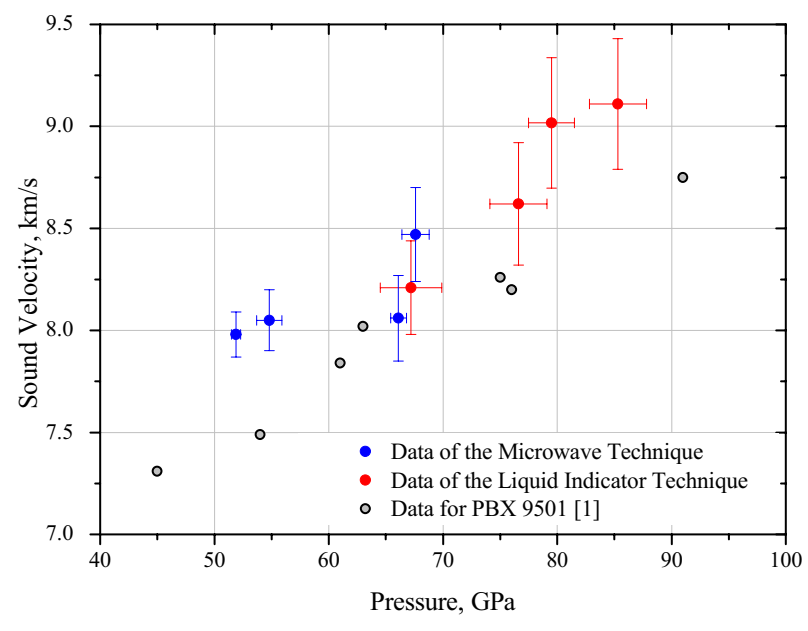

- data obtained by using a radio interferometer; - - data obtained by indicator liquid technique; - data of the USA for the composition PBX 9501 [1].

Figure 6. Dependence of sound velocities on pressure in overcompressed explosion products.

products in this case by the formula:

$$
C_{C}=\frac{X_{M A X}\left(D_{C}-U_{C}\right)}{D_{C} \cdot\left(T_{1}-T_{2}+\frac{X_{M A X}}{D_{C}}\right)},
$$

where $T_{1}$ is the time of a shock wave entering the sample; $T_{2}$ is the time of rarefaction wave entering the interface between a screen and $\mathrm{HE}$; $D_{C}$ is the velocity of a detonation wave in a sample; $U_{C}$ is the mass velocity behind a detonation front; $X_{M A X}$ is the thickness of a step, when overtaking occurs at the sample/indicator interface.

The values $T_{1}$ and $T_{2}$ are defined by the help of the $X-t$ diagram of Fig. 2. The calculations were made with the use of reference data from [8] in the gasdynamic code "UP" [9], in which equations of state of materials are given in the form of Mie-Grueneisen. 
Table 1. Sound velocity in overcompressed explosion products (radiowave technique).

\begin{tabular}{|c|c|c|c|c|c|c|c|}
\hline Test num & $\rho_{0}, \mathrm{~g} / \mathrm{cm}^{3}$ & $W, \mathrm{~km} / \mathrm{s}$ & $D_{c}, \mathrm{~km} / \mathrm{s}$ & $U, \mathrm{~km} / \mathrm{s}$ & $P, \mathrm{GPa}$ & $T, \mu \mathrm{s}$ & $C_{c}, \mathrm{~km} / \mathrm{s}$ \\
\hline 1 & 1,885 & $5,22 \pm 0,03$ & $9,35 \pm 0,04$ & $2,95 \pm 0,03$ & $51,99 \pm 0,40$ & $2,02 \pm 0,02$ & $7,98 \pm 0,11$ \\
\hline 2 & 1,886 & $5,45 \pm 0,09$ & $9,42 \pm 0,03$ & $3,08 \pm 0,07$ & $54,72 \pm 1,10$ & $2,81 \pm 0,02$ & $8,05 \pm 0,15$ \\
\hline 3 & 1,885 & $4,96 \pm 0,04$ & $9,80 \pm 0,07$ & $3,58 \pm 0,04$ & $66,13 \pm 0,69$ & $1,89 \pm 0,06$ & $8,06 \pm 0,21$ \\
\hline 4 & 1,885 & $4,96 \pm 0,04$ & $10,02 \pm 0,13$ & $3,58 \pm 0,04$ & $67,62 \pm 1,19$ & $2,53 \pm 0,13$ & $8,47 \pm 0,23$ \\
\hline
\end{tabular}

Where $\rho_{0}$ is the initial density of HE samples; $W$ is the impactor velocity; $D_{c}$ is the velocity of an overcompressed detonation wave in HE; $U$ is the mass velocity behind a front of an overcompressed detonation wave in HE; $P$ is the pressure of an overcompressed detonation wave in HE; $T$ is the time of the arrival of a lateral rarefaction wave at a detonation front; $C_{c}$ is the sound velocity in overcompressed explosion products of the composition.

Table 2. Sound velocity in overcompressed explosion products (indicator liquid technique).

\begin{tabular}{|c|c|c|c|c|c|c|c|c|c|}
\hline Test num & $\rho_{0}, \mathrm{~g} / \mathrm{cm}^{3}$ & $W, \mathrm{~km} / \mathrm{s}$ & $T_{1}, \mu \mathrm{s}$ & $T_{2}, \mu \mathrm{s}$ & $X_{M A X}, \mathrm{~mm}$ & $D_{c}, \mathrm{~km} / \mathrm{s}$ & $U, \mathrm{~km} / \mathrm{s}$ & $P, \mathrm{GPa}$ & $C_{c}, \mathrm{~km} / \mathrm{s}$ \\
\hline 1 & 1,882 & $4,94 \pm 0,04$ & 0,289 & 0,644 & 10,7 & 9,43 & 3,78 & 67,2 & 8,21 \\
\hline 2 & 1,887 & $4,94 \pm 0,04$ & 0,278 & 0,635 & 11,1 & 9,95 & 4,09 & 76,6 & 8,62 \\
\hline 3 & 1,880 & $5,00 \pm 0,05$ & 0,286 & 0,648 & 10,5 & 10,07 & 4,18 & 79,5 & 9,02 \\
\hline 4 & 1,887 & $5,58 \pm 0,06$ & 0,162 & 0,410 & 7,68 & 10,40 & 4,35 & 85,3 & 9,11 \\
\hline
\end{tabular}

Where $T_{1}$ is the time of a shock wave entering the sample; $T_{2}$ is the time of rarefaction wave entering the interface between a screen and HE; $X_{M A X}$ is the thickness of a step, when overtaking occurs at the sample/indicator interface.

\section{Experimental setup}

To generate over-compressed detonation waves in samples of HMX-based explosives, the experts used plane shock wave generators and cylindrical active HE charges of diameter $120 \mathrm{~mm}$ based on RDX and HMX with different power; their explosion products were accelerated by aluminum (AD-1) impactors or steel (St 3) impactors. Sketches of experimental assemblies are given in Fig. 3.

The values of the flying up impactor velocity $W$ were determined in experiments as per a scheme of Fig. 3 (left) with the use of a radiowave technique. The authors determined also the velocities of the overcompressed detonation wave $D_{C}$ and the time $T$ of overtaking of a detonation front by a rarefaction wave using a radiowave technique. Figure 4 presents a typical experimental dependence of a detonation wave velocity on time.

The HE samples under study were placed into a capsule, whose construction consists of: a screen, a technological composite cartridge and a cap with outputs for optical fiber and electrocontact pins in experiments with the use of the optical method (Fig. 3, right). Four blind holes ("wells") of diameter $16 \mathrm{~mm}$ were milled in the HE sample $60 \mathrm{~mm}$ in diameter. Each "well" had its depth, namely, a thickness of a bottom - a step of a sample. "Wells" were filled with indicator liquid. Carbogal (perfluor-1.3-dimethylcyclohexane, the initial density $\rho_{0}=1.856 \mathrm{~g} / \mathrm{cm}^{3}$ ) was used as indicator liquid in experiments. Photodiode sensors recorded luminescence of an indicator via optical outputs and quartz-polymer fibers located above each well. In this case, walls of the "wells" excluded lateral flare from adjoining sample steps. Electrocontact pins and optical sensors diagnosed state parameters in an explosive in the experiment. The accuracy of time measurements of motion of shock wave front in liquid indicators is not worse than 2 ns. Figure 5 presents one of the oscillograms of a test, recorded by the help of a photodiode sensor.

By using indicator liquid, a relative error of measurements of sound velocities by the help of the optical method does not exceed $7 \%$.

\section{Experimental results}

The data obtained in tests for measurements of sound velocities in overcompressed explosion products of the explosive composition being studied are tabulated (Tables 1 and 2).

Figure 6 presents the dependence of the obtained sound velocities in explosion products of the composition under study on a pressure in overcompressed detonation front. Besides, the graph contains the data for sound velocities in the composition PBX 9501 from [1].

As is possible to see in the graph of Fig. 6, the data on sound velocities in explosion products of the composition under study, which were obtained by two different techniques independently from each other, are in agreement with one another and they do not contradict the data for PBX 9501 with the similar HE composition from work [1].

\section{Conclusion}

By using the overtaking unloading method, sound velocities were determined through radiowave and optical techniques in explosion products of the HMX-based composition, which were overcompressed up to 50 $85 \mathrm{GPa}$. The data on sound velocities in explosion products of the composition under study, which were obtained by two different methods independently from each other, are in agreement with one another and with the data for a foreign analog PBX 9501. The achieved results testify to the fact that techniques with the use of a radiointerferometer and indicator liquid, are usable for determination of sound velocities in overcompressed explosion products and for research of parameters at the Jouget point of various HEs.

\section{References}

[1] J.N. Fritz, R.S. Hixson, M.S. Shaw, C.E. Morris, and R.G. McQueen, J. Appl. Phys. 80, 11 (1996)

[2] V.P. Chelyshev, B.I. Shekhter, FGV, 4, p.68-74 (1966) 
[3] A.L. Mikhaylov, V.E. Kostyukov, Yu.I. Orekhov et al., Proceedings of International Conference "VII Khariton's Topical Scientific Readings. Extreme states of substance. Detonation. Shock waves", Sarov, RFNC-VNIIEF, p.649-654 (2005)

[4] A.V. Rodionov, V.M. Bel'skiy, E.N. Bogdanov et al., Proceedings of International Conference "XI Khariton's Topical Scientific Readings. Extreme states of substance. Detonation. Shock waves", Sarov, RFNC-VNIIEF, p.69-73 (2009)

[5] V.M. Bel'skiy, A.L. Mikhaylov et al., Proceedings of International Conference "XIII Khariton's Topical Scientific Readings. Extreme states of substance. Detonation. Shock waves", Sarov, RFNC-VNIIEF, p.785-794 (2011)
[6] R.G. McQueen, J.W. Hopson, J.N. Fritz, Rev. Sci. Instrum, 53, 2, p.245-250 (1982)

[7] M.V. Zhernokletov, A.E. Kovalev, V.V. Komissarov et al., SCCM-2007, ed. by M.L. Elert, M.D. Furnish, R. Chau, N. Holmes, J. Nguyen. - AIP, p.117-120 (2007)

[8] R.F. Trunin, Experimental data on shock-wave compression and adiabatic expansion of condensed materials, RFNC- VNIIEF, Sarov, p.531 (2006)

[9] N.F. Gavrilov, G.G. Ivanova, V.I. Selin, V.N. Sofronov, Problems of atomic science and engineering. Series: Methods and codes for numerical solution of problems of mathematical physics, 3, 11, p.11-14 (1982) 